The Astrophysical Journal, 633:452-459, 2005 November 1

(C) 2005. The American Astronomical Society. All rights reserved. Printed in U.S.A.

\title{
MULTIPLICITY AT THE STELLAR/SUBSTELLAR BOUNDARY IN UPPER SCORPIUS
}

\author{
Adam L. Kraus, Russel J. White, and Lynne A. Hillenbrand \\ Department of Astrophysics, California Institute of Technology, MC 105-24, Pasadena, CA 91125; \\ alk@astro.caltech.edu, rjw@astro.caltech.edu, lah@astro.caltech.edu \\ Received 2005 March 11; accepted 2005 July 11
}

\begin{abstract}
We present the results of a high-resolution imaging survey of 12 brown dwarfs and very low mass stars in the closest ( $\sim 145 \mathrm{pc})$ young ( $\sim 5 \mathrm{Myr})$ OB association, Upper Scorpius. We obtained images with the Advanced Camera for Surveys High Resolution Channel on HST through the F555W $(V)$, F775W $\left(i^{\prime}\right)$, and F850LP $\left(z^{\prime}\right)$ filters. This survey discovered three new binary systems, including one marginally resolved pair with a projected separation of only $4.9 \mathrm{AU}$, resulting in an observed binary fraction of $25 \% \pm 14 \%$ at separations $\gtrsim 4 \mathrm{AU}$. After correcting for detection biases assuming a uniform distribution of mass ratios for $m_{s} / m_{p}>0.6$, the estimated binary fraction is $33 \% \pm 17 \%$. The binary fraction is consistent with that inferred for higher mass stars in Upper Sco, but the separation and mass ratio distributions appear to be different. All three low-mass binary systems in Upper Sco are tight $(<18 \mathrm{AU})$ and of similar mass $\left(m_{s} / m_{p} \gtrsim 0.6\right)$, consistent with expectations based on previous multiplicity studies of brown dwarfs and very low mass stars in the field and in open clusters. The implication is that the distinct separation and mass ratio distributions of low-mass systems are set in the formation process or at very young ages, rather than by dynamical disruption of wide systems at ages $\gtrsim 5$ Myr. Finally, we combine the survey detection limits with low-mass evolutionary models to show that there are no planets or very low mass brown dwarfs with masses $>10 M_{\mathrm{J}}$ at projected separations $>20 \mathrm{AU}$ or masses $>5 M_{\mathrm{J}}$ at projected separations $>40 \mathrm{AU}$ orbiting any of the low-mass $\left(0.04-0.10 M_{\odot}\right)$ objects in our sample.
\end{abstract}

Subject headings: binaries: visual — stars: low-mass, brown dwarfs — stars: pre-main-sequence

\section{INTRODUCTION}

Brown dwarfs (BDs) are objects with masses intermediate between those of stars and planets. As such, studies of BDs potentially can offer a unique link between theories of star formation and planet formation. Several BD formation scenarios have been proposed, but current observational results indicate that the properties of BDs, including their spatial distribution in star-forming regions (Luhman 2004b; Briceno et al. 2002), velocity dispersion (Joergens \& Guenther 2001; White \& Basri 2003), accretion and inner disk frequency (Muench et al. 2001; Jayawardhana et al. 2003; Liu et al. 2003; Mohanty et al. 2005), and evidence for an embedded phase (White \& Hillenbrand 2004), are consistent with those of low-mass stars. Although this strongly supports the idea that BDs form in a fashion similar to stars, via the dynamical collapse and fragmentation of a cloud core, there is emerging evidence that the binary properties of BDs are fundamentally different from those of low-mass stars.

Multiplicity surveys of field T dwarfs (Burgasser et al. 2003), L dwarfs (Koerner et al. 1999; Reid et al. 2001; Close et al. 2003; Bouy et al. 2003; Gizis et al. 2003), late M dwarfs (Siegler et al. 2005), and intermediate-age BDs in the Pleiades (Martin et al. 2000) have found lower binary frequencies and smaller binary separations than for field G and early M dwarfs (Duquennoy \& Mayor 1991; Fischer \& Marcy 1992). It is unclear whether these binary properties reflect differences in the formation mechanism or the dynamical disruption of wide substellar binaries. One way to distinguish between these scenarios is by studying the properties of young BD binaries, possibly before any significant dynamical evolution has occurred. In this paper we present the results of an imaging multiplicity survey of BDs in the nearest $(\sim 145 \mathrm{pc})$ young ( $\sim 5$ Myr; Preibisch \& Zinnecker 1999) OB association, Upper Sco.

\section{OBSERVATIONS AND DATA REDUCTION}

Our targets were selected from the survey for low-mass members of Upper Sco by Ardila et al. (2000). They identified 15 candidate members with spectral types of M5.5 or cooler, based on either low-resolution spectra (10 objects) or $R-I_{\mathrm{C}}$ colors (five objects). Three of these 15 (USco 85, 114, 121) were determined to be likely nonmembers, based on low lithium abundance and radial velocities that are inconsistent with higher mass members (Muzerolle et al. 2003; R. J. White et al. 2005, in preparation). The remaining 12 were observed in this program. Subsequently, membership for all but the faintest member of this sample, USco 137 , has been confirmed with additional high-resolution spectra (Mohanty et al. 2005). USco 137 has not yet been observed spectroscopically. Since our targets include both very low mass stars and BDs, we hereafter refer to them as very low mass objects, or VLMOs.

Our images were obtained with the Advanced Camera for Surveys High Resolution Channel on the Hubble Space Telescope, which has a field of view of $26 \times 29$ and a distortion-corrected pixel size of 27 mas pixel $^{-1}$. Observations were made between July and September 2003 with the filters F555W (V), F775W $\left(i^{\prime}\right)$, and F850LP $\left(z^{\prime}\right)$ at two dither positions near the center of the detector and with two exposures per position. Total integration times were 510, 300, and $200 \mathrm{~s}$, respectively. The F555W exposure times for the brightest objects (USco-55, 66, 67, and 75) were reduced to $350 \mathrm{~s}$ to allow for additional short exposures in F775W and F850LP, which were close to the saturation limit in the full-length exposures. We chose the $V$ band to maximize angular resolution (diffraction limit $\theta_{\text {res }, V}=58 \mathrm{mas}$ ) and the $i^{\prime}$ and $z^{\prime}$ bands to maximize sensitivity to very low mass companions.

The raw images were calibrated and distortion-corrected by the CALACS pipeline during on-the-fly reprocessing (Mack et al. 2003). Some cosmic rays remained, but their morphologies were 
TABLE 1

Very Low Mass Objects in Upper Sco

\begin{tabular}{|c|c|c|c|c|c|c|}
\hline Name & Date $^{\mathrm{a}}$ & $V^{\mathrm{b}}$ & $i^{\prime \mathrm{b}}$ & $z^{\prime b}$ & $\mathrm{SpT}^{\mathrm{c}}$ & $\begin{array}{c}M^{\mathrm{d}} \\
\left(M_{\odot}\right)\end{array}$ \\
\hline USco-55 А ………………........ & 49.9 & $18.73 \pm 0.04$ & $15.92 \pm 0.012$ & $14.81 \pm 0.09$ & M5.5 & $0.10 \pm 0.03$ \\
\hline 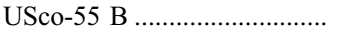 & 49.9 & $18.92 \pm 0.015$ & $16.19 \pm 0.08$ & $15.12 \pm 0.09$ & (M6.0) & $0.07 \pm 0.02$ \\
\hline 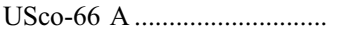 & 62.6 & $18.92 \pm 0.021$ & $16.36 \pm 0.016$ & $15.41 \pm 0.012$ & M6.0 & $0.07 \pm 0.02$ \\
\hline 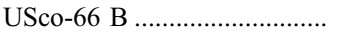 & 62.6 & $18.94 \pm 0.022$ & $16.30 \pm 0.023$ & $15.29 \pm 0.008$ & (M6.0) & $0.07 \pm 0.02$ \\
\hline USco-67 ........................................... & 49.0 & $18.47 \pm 0.002$ & $15.52 \pm 0.001$ & $14.32 \pm 0.001$ & M5.5 & $0.10 \pm 0.03$ \\
\hline 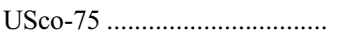 & 59.7 & $18.71 \pm 0.002$ & $15.80 \pm 0.001$ & $14.61 \pm 0.001$ & M6.0 & $0.07 \pm 0.02$ \\
\hline 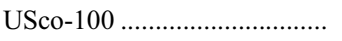 & 67.0 & $19.09 \pm 0.002$ & $15.99 \pm 0.001$ & $14.73 \pm 0.001$ & M7.0 & $0.05 \pm 0.01$ \\
\hline 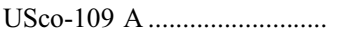 & 64.6 & $20.20 \pm 0.03$ & $17.13 \pm 0.04$ & $15.87 \pm 0.016$ & M6.0 & $0.07 \pm 0.02$ \\
\hline USco-109 В …………............... & 64.6 & $21.21 \pm 0.076$ & $18.07 \pm 0.08$ & $16.92 \pm 0.04$ & (M7.5) & $0.04 \pm 0.01$ \\
\hline 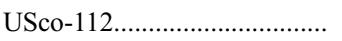 & 71.6 & $18.04 \pm 0.001^{\mathrm{e}}$ & $16.15 \pm 0.001$ & $15.17 \pm 0.001$ & M5.5 & $0.10 \pm 0.03$ \\
\hline USco-128 & 70.6 & $21.29 \pm 0.007$ & $17.79 \pm 0.002$ & $16.34 \pm 0.002$ & M7.0 & $0.05 \pm 0.01$ \\
\hline USco-130 ………………........ & 87.8 & $21.39 \pm 0.008$ & $17.80 \pm 0.002$ & $16.30 \pm 0.002$ & $\mathrm{M} 7.5^{\mathrm{f}}$ & $0.04 \pm 0.01$ \\
\hline 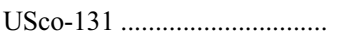 & 63.6 & $21.98 \pm 0.011$ & $18.22 \pm 0.002$ & $16.67 \pm 0.002$ & $\mathrm{M} 6.5^{\mathrm{f}}$ & $0.06 \pm 0.01$ \\
\hline 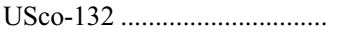 & 88.8 & $22.11 \pm 0.013$ & $18.31 \pm 0.002$ & $16.59 \pm 0.002$ & M7.0 & $0.05 \pm 0.01$ \\
\hline 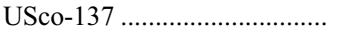 & 88.9 & $22.92 \pm 0.023$ & $19.27 \pm 0.004$ & $17.75 \pm 0.003$ & M7.0 $0^{\mathrm{f}}$ & $0.05 \pm 0.01$ \\
\hline
\end{tabular}

a Observation date: JD-2,452,800.

${ }^{\mathrm{b}}$ Uncertainties are statistical; systematic uncertainties due to aperture corrections and conversion to standard systems are $\sim 0.03$ mag.

${ }^{c}$ Spectral types in parentheses are inferred from photometry presented here; others are from Ardila et al. (2000; $\left.\S 4.1\right)$.

d Masses are determined from the models of Baraffe et al. (1998).

e The $V$ magnitude for USco-112 is anomalously bright ( $(2)$.

${ }^{\mathrm{f}}$ Spectral type is based only on photometric $R-I_{\mathrm{C}}$ colors.

substantially different from stellar point-spread functions (PSFs), so they were easily identified by visual inspection.

Potential point sources were identified with the IRAF task DAOPHOT/DAOFIND, which found all local brightness maxima with a significance of $>5 \sigma$ and a FWHM near the expected value for the filter. We then measured aperture photometry and PSF-fitting photometry for all objects in each field, and we report aperture photometry for all isolated objects and PSF photometry for all close binaries. PSF magnitudes were corrected to match aperture magnitudes based on results for the nine well-sampled objects that appeared isolated under visual inspection. Aperture photometry was carried out with the IRAF task DAOPHOT/ PHOT with a 5 pixel aperture for faint objects and a 10 pixel aperture for bright objects, and our sky annulus had an inner radius of 200 pixels and a width of 15 pixels. We used the finite aperture corrections of Sirianni et al. (2005). PSF-fitting photometry was carried out with the IRAF task DAOPHOT/ALLSTAR (Stetson 1987). The PSF for each filter was constructed from the nine isolated VLMOs. Since all of the targets were located near the center of the chip and have similar temperatures and extinctions, location and color effects should not be important. There was some variation in the PSF FWHM from target to target $( \pm 5 \%)$, which we attribute to small orbit-to-orbit changes in focus. However, the radial shape of the PSF was very similar for all objects except USco-109, which we discuss in more detail in $\S 3.1$.

Transformations to ground-based magnitudes $\left(V\right.$, SDSS $i^{\prime}$, and SDSS $z^{\prime}$ ) were calculated with the IRAF task SYNPHOT/ CALCPHOT, which convolves an input spectrum with transmission curves for the Hubble Space Telescope (HST) optics and filters or standard ground-based filters. Since SYNPHOT does not include transmission curves for the $i^{\prime}$ and $z^{\prime}$ filters, which are defined at the United States Naval Observatory 40 inch $(1 \mathrm{~m})$ telescope, they were obtained from the Web site for the Sloan Digital Sky Survey ${ }^{1}$ (SDSS) and represent the filters and optics at the USNO 40 inch $(1 \mathrm{~m})$ telescope observing at 1.3 air masses

\footnotetext{
${ }^{1}$ See http://www.sdss.org/dr1/algorithms/standardstars/Filters/response.html.
}

(Fukugawa et al. 1996). Based on transformations determined for a set of M0-M8 dwarfs and M5-M8 giants from the BruzualPersson-Gunn-Stryker Spectrophotometry Atlas (Bruzual et al. 1996), we find constant corrections that do not depend significantly on temperature or surface gravity: $m_{555}-m_{V}=-0.16 \pm$ $0.03, m_{775}-m_{i^{\prime}}=+0.07 \pm 0.03$, and $m_{850}-m_{z^{\prime}}=+0.03 \pm$ 0.03 . The uncertainties are estimated from the standard deviations of all tested objects. The transformed magnitudes ${ }^{2}$ are listed in Table 1. The statistical uncertainties correspond to either the photon noise (for aperture photometry; typically $<0.01 \mathrm{mag}$ ) or the goodness of fit (for PSF-fitting photometry; 0.02-0.09 mag). Systematic uncertainties in the magnitude transformations and aperture corrections are $\sim 0.03 \mathrm{mag}$.

\section{RESULTS}

\subsection{New VLMO Binaries}

In Figure 1, we present contour plots of three candidate binaries (USco-55, 66, and 109) and the apparently single star USco-67 in the F555W, F775W, and F850LP filters. The USco-55 and USco-66 systems are clearly resolved. The USco-109 system is not obviously resolved and was not initially reported as a double source by ALLSTAR, but the PSF appears to be marginally elongated in the positive $x$-direction relative to the single VLMO USco-67.

One limitation in the ALLSTAR-based data reduction method is that binaries with very close $(\lesssim \lambda / D)$ separations are often not identified, even when their combined PSF is elongated at a high confidence level. DAOFIND, the task that identifies potential objects in the images, only identifies point sources based on the

2 The $V$-band flux for U Sco-112 appears to be anomalously bright. Based on its $i^{\prime}$ and $z^{\prime}$ magnitudes, it appears to be of brightness and color similar to USco-55 B. However, it is $0.88 \pm 0.06$ mag brighter than USco-55 B in $V$. Exposures in both dither positions give consistent fluxes, so it is probably not the result of a cosmic-ray hit on one exposure. We suggest that this is caused either by a transient optical brightening of the system (e.g., a flare or increased accretion) or by contamination from a spatially unresolved blue background object. 


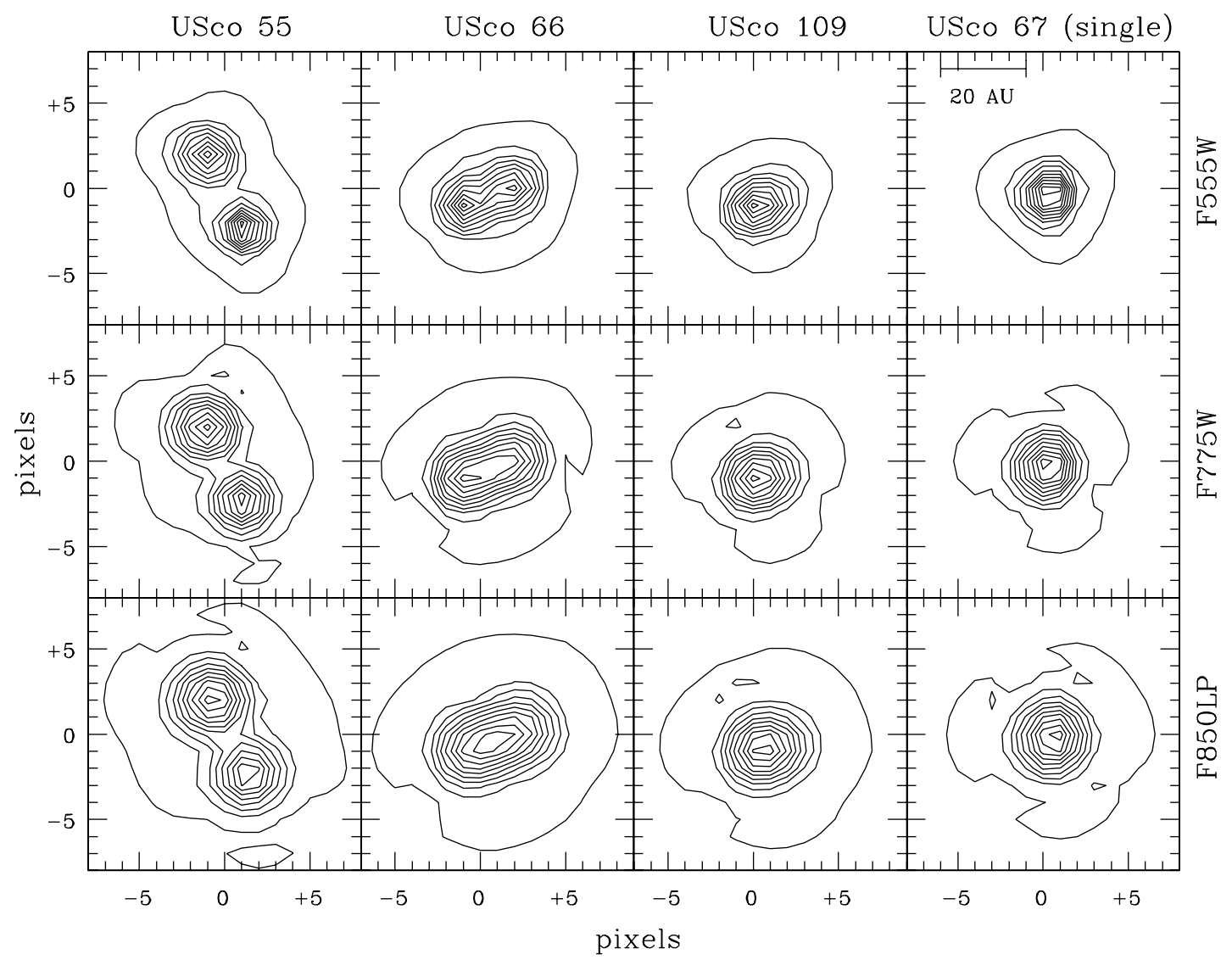

Fig. 1. - Left to right, Contour plots of four targets: USco-55, USco-66, USco-109, and USco-67. Units are pixels, and the projected physical scale at the distance of Upper Sco is shown in the upper right panel. Contours are drawn at $95 \%$ through $5 \%$ of the maximum pixel value, in increments of $10 \%$. The field of view in each image is 432 mas, or $\sim 60 \mathrm{AU}$ at $145 \mathrm{pc}$.

presence of a distinct peak. Thus, automated photometry is biased against the detection of very close binaries. This limitation can be overcome for known or suspected binaries by manually adding a second point source in approximately the correct location and letting ALLSTAR recenter it to optimize the fit; if it does not produce a statistically significant fit, ALLSTAR then discards it. We have done this for USco-109.

In Figure 2, we present plots for USco-109 and the next brightest object in the same images, a likely background field star $(\S 3.2)$. The first three columns show USco-109 and the residuals from fitting with one and then two point sources, and the last two columns show the field star and its single-source fit. The maximum and minimum pixel values are also given to allow quantitative comparison of the residuals to the original images. The common position angle of the residuals in the single-source fit in all three filters seems to imply that this elongation is a real effect, and not simply noise. USco-109 appears elongated in the same direction relative to both its neighbor and USco-67; this extension is therefore unlikely to be an artifact due to excess jitter, which would affect the field star as well. USco-109 is better fit with two point sources in all three filters, and the fit reduces the residuals by factors of $2-8$. Moreover, these fits independently find similar positions and flux ratios in all three filters, which further supports its classification as a binary system. Since the similar two-source residuals in each filter imply some remaining uncertainty in the fit and the separation is only $\sim 1.3$ pixels, follow-up observation of this system to confirm its multiplicity and properties should be a priority. However, we proceed under the assumption that it is a binary system in our subsequent analysis.
We summarize the properties of these three systems in Table 2. The uncertainties in separation and position angle are determined from the standard deviations in the locations as reported by ALLSTAR for the three filters.

\subsection{VLMOs and Background Stars}

In Figure 3, we present an $i^{\prime}$ versus $i^{\prime}-z^{\prime}$ color-magnitude diagram of the 12 primary targets (filled circles) and all other objects detected at the $5 \sigma$ level in both filters. Objects within $5^{\prime \prime}(800 \mathrm{AU})$, which are statistically more likely to be physically associated, are shown as open circles, while other objects are shown as crosses. Also shown are the average main sequence at the distance of Upper Sco (Hawley et al. 2002) and a 5 Myr isochrone based on the evolutionary models of Baraffe et al. (1998). The location of the isochrone is determined by converting the predicted $I_{\mathrm{C}}$ and $J$ magnitudes to $i^{\prime}$ and $z^{\prime}$ magnitudes, using $I_{\mathrm{C}}-i^{\prime}$ colors derived using the methods described in $\S 2$ and $z^{\prime}-J$ colors found from the SDSS field main sequence for older, more massive M dwarfs by Hawley et al. (2002). Although the $I_{\mathrm{C}}-i^{\prime}$ transformations should be accurate $(\S 2)$, the $z^{\prime}-J$ transformations may be more sensitive to surface gravity differences due to the larger difference in central wavelengths. Consequently, the $i^{\prime}-z^{\prime}$ color of the $5 \mathrm{Myr}$ isochrone is somewhat uncertain.

The three very close companions to USco-55, USco-66, and USco-109 are located above the SDSS main sequence and well above the background population, so they are most likely association members. Based on their close proximity and the low density of association members, we conclude that these are physically associated companions. All other objects fall well below 
USco 109 Single Fit Double Fit Field Star Single Fit

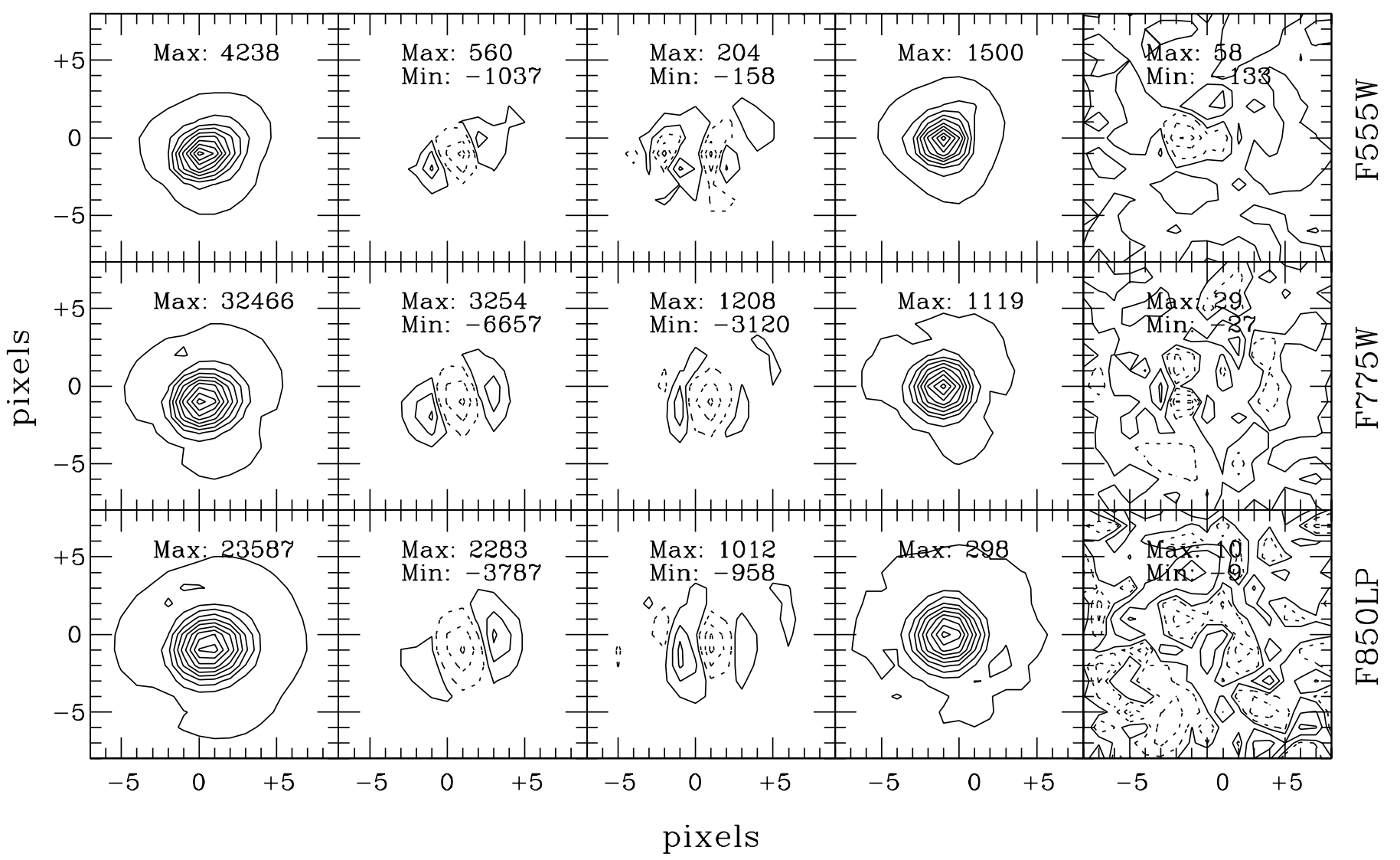

FIG. 2.- Contour plots of USco-109 and a background star in the same field for all three filters. The first three columns show USco-109 and the residuals from fitting with one and then two point sources, and the last two columns show the background star and the residuals from a single-source fit. For residuals, contours are drawn at the $90 \%, 50 \%$, and 10\% levels of maximum (solid lines) and minimum (dashed lines). The maximum and minimum pixel values are given to allow comparison of the residuals to the original images. The pixel values in the last column, where the sky background contours fill each panel and obscure the labels, are $(58,-133)$, $(29,-27)$, and $(10,-9)$, respectively.

the empirical main sequence and are most likely background stars. The marginally resolved companion to USco-109 resides near the empirical (but somewhat uncertain) main sequence, but given the uncertainty in its $i^{\prime}-z^{\prime}$ color, its location is consistent with the broad luminosity distribution of low-mass Upper Sco members that we discuss in $\S 5$.

\subsection{Sensitivity Limits}

We determined detection limits as a function of distance from the primary stars via a Monte Carlo simulation similar to that of Metchev et al. (2003). We used the IRAF task DAOPHOT/ ADDSTAR and our average PSF to add artificial stars at a range of radial separations and magnitudes to the fields of USco-67, USco-128, and USco-137, which represent the maximum, median, and minimum brightness sources in our sample. We then attempted to identify them with our ALLSTAR photometry pipe- line. In Figure 4, we show the detection limits for the USco-67 and USco-128 fields in $V$ and $z^{\prime}$, as a function of the separation at which we can detect $>10 \%, 50 \%$, and $90 \%$ of the companions. At small separations ( $\leqslant 20 \mathrm{AU}$ ), the $50 \%$ detection thresholds roughly scale with the brightness of the primary; they are similar for all objects in terms of $\Delta m$. The detection limits converge to constant values at large separations at which the background dominates the noise: $z^{\prime}=23.40 \pm 0.05, i^{\prime}=25.08 \pm 0.05$, and $V=26.01 \pm 0.05$ (uncertainties are based on the standard deviation between the three fields). The simulations demonstrate that we potentially could identify bright pairs as close at 1 pixel $(0.025 ; 4 \mathrm{AU})$ but none closer. We also show the locations (in $\Delta m$ and separation) of the three companions to USco-55, 66, and 109. These results indicate that the probability of detecting a system with the separation and flux ratio of USco-109 with automated photometry is between $10 \%$ and $50 \%$ for the brightest

TABLE 2

Binary Parameters

\begin{tabular}{cccccc}
\hline \hline Name & $\begin{array}{c}\text { Projected Separation } \\
(\mathrm{mas})\end{array}$ & $\begin{array}{c}\text { Projected Separation } \\
(\mathrm{AU})\end{array}$ & $\begin{array}{c}\text { Position Angle } \\
(\text { deg})\end{array}$ & $\Delta \mathrm{SpT}$ & $q$ \\
\hline USco-55 AB …............. & $121.6 \pm 0.6$ & $17.63 \pm 0.09$ & $307.7 \pm 0.4$ & $0.5 \pm 0.1$ & $0.86 \pm 0.04$ \\
USco-66 AB ................. & $70.3 \pm 0.5$ & $10.19 \pm 0.07$ & $31.7 \pm 0.2$ & $0.0 \pm 0.1$ & $0.94 \pm 0.03$ \\
USco-109 AB ............... & $34 \pm 2$ & $4.9 \pm 0.3$ & $302 \pm 3$ & $1.5 \pm 0.2$ & $0.59 \pm 0.04$ \\
\hline
\end{tabular}




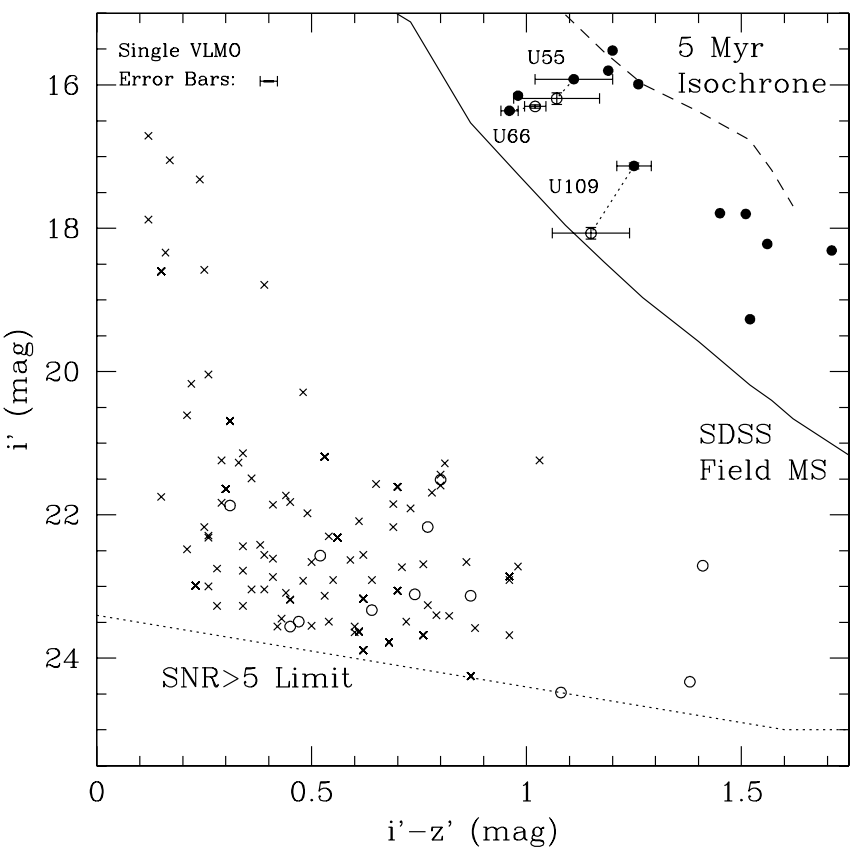

FIG. 3.-An $i^{\prime}$ vs. $i^{\prime}-z^{\prime}$ color-magnitude diagram. Primary Upper Sco targets are shown as filled circles, neighbors within $5^{\prime \prime}$ are shown as open circles, and widely separated objects are shown as crosses. The SDSS field main sequence (solid line), a 5 Myr isochrone (dashed line), and the detection limits of the survey (dotted line) are also shown. Candidate binary VLMO pairs are connected with dotted lines and labeled. The error bars for binary components are associated with each point, and the error bars for single objects are shown in the upper left corner. objects and becomes negligible for the fainter targets in our sample. However, as discussed in $\S 2$, these limits are conservative at small separations, since ALLSTAR often reports only a single point source when the PSF is still noticeably elongated and manual-fitting can distinguish two point sources. The position angle of the binary can also play a role in the detection probability; as can be seen in Figure 1, the blended PSF of USco-109 is elongated along the axis where the PSF is narrowest, providing the most significant possible detection. Artificial star tests show that if the companion was near one of the extended lobes of the PSF, it would have been much more difficult to identify.

\subsection{Uncertainties in Binary Properties}

A similar Monte Carlo routine was used to test the uncertainties in the measurements of USco-109. We used ADDSTAR and the average PSF to construct 100 simulated images, given the positions and brightnesses reported for the primary and the secondary for the real images, and then used ALLSTAR to perform PSF-fitting photometry on these simulated images. The standard deviation in separation ( $\sim 1$ mas) from the simulated images is consistent with that calculated from the standard deviation in separation between the three filters $(\sim 2$ mas), and the standard deviation in the flux ratio $\Delta m\left(0.09 \mathrm{mag}\right.$ in $V, 0.06$ in $i^{\prime}$ and $\left.z^{\prime}\right)$ is consistent with that determined from the magnitudes reported by ALLSTAR for each object.

\section{ANALYSIS}

\subsection{Inferred Properties}

In Table 1, we give the inferred spectral types and masses for all of the VLMOs in our sample. Spectral types for single

USco 67

USco 128

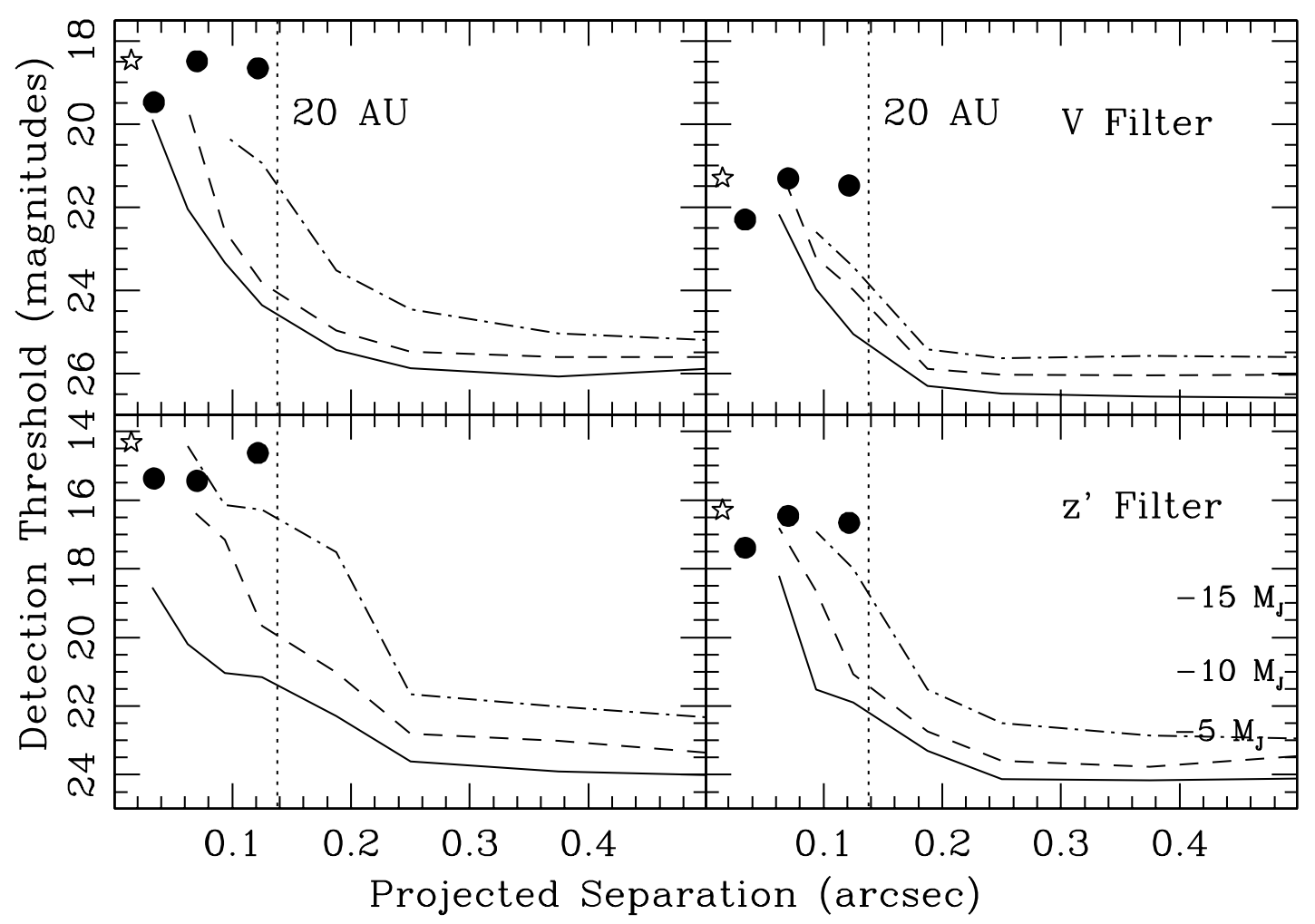

FIG. 4.-Detection frequencies at $5 \sigma(10 \%$, solid line; $50 \%$, dashed line; $90 \%$, dash-dotted line $)$ as a function of separation in each filter for the VLMOs of maximum and median brightness, USco-67 and USco-128. Corresponding brightnesses of potential planetary-mass companions are shown on the right for the $z^{\prime}$ plot. The brightness of the primary object is denoted with a star to allow conversion to $\Delta m$ values, and the vertical dotted line indicates a separation of $20 \mathrm{AU}$ at the distance of Upper Sco (145 pc). The filled circles mark the separation and $\Delta m$ values for the three binary systems. 
VLMOs and for binary primaries in our sample are taken from Ardila et al. (2000). Spectral types for nine objects were determined from low-resolution spectra; the other three (USco-130, 131 , and 137) have only photometric spectral types as determined from $R-I_{\mathrm{C}}$ colors. Ardila et al. (2000) found that although this region is young, the extinction is generally low $\left(A_{V}<1\right)$, so the photometric spectral types should be reasonably accurate. Spectroscopic observations of all targets except USco-137 by Mohanty et al. (2005) appear to be consistent with these assigned spectral types.

The masses for this sample are estimated from the 5 Myr massmagnitude-temperature relations of Baraffe et al. (1998) and the temperature-SpT relations of Luhman et al. (2003) and range from 0.04 to $0.10 M_{\odot}$. Large systematic errors may be present in these and all pre-main-sequence models (e.g., Baraffe et al. 2002; Close et al. 2005; Reiners et al. 2005), so they are best used for comparison only. The models of Baraffe et al. predict that the mass ratio $\left(q=m_{s} / m_{p}\right)$ and $\Delta$ SpT for the three binaries are a function of the primary-to-secondary flux ratios $\Delta m$, with only a minor mass dependence; we report these quantities as determined from the flux ratio $\Delta i^{\prime}$ in Table 1 . The uncertainties reflect the uncertainties in the flux ratios but do not include any systematic uncertainties from the models. The values determined from $\Delta V$ and $\Delta z^{\prime}$ are consistent with these results, but the values for $\Delta i^{\prime}$ are least dependent on the primary mass, so they are most reliable.

\subsection{The Binary Frequency at the Stellar/Substellar Boundary}

For our 12 M5.5-M7.5 targets, we have detected three binary VLMO systems with projected separations of 4.9-17.6 AU, giving an observed binary fraction of $25 \% \pm 14 \%$. However, as can be seen in Figure 4, the detection of faint companions is difficult at separations comparable to the PSF width (58 mas in V). Consequently, the total binary fraction may be higher. We can estimate the number of undetected companions via a method based on that of Close et al. (2003), in which we convolve an assumed mass ratio distribution with the mass-magnitude relations of Baraffe et al. (1998) and the detection thresholds $\Delta m$ as calculated in $\S 4$ 4.3. Similar results are found for all three filters; the following results are those for the $z^{\prime}$ band.

Observations of field $\mathrm{M}$ dwarfs have found a flat mass ratio distribution from 1.0 to 0.1 (Fischer \& Marcy 1992) for all companions at separations $a>4$ AU. However, studies of field VLMOs have found only $\mathrm{BD}$ companions with mass ratios $q>$ $0.6(\Delta m<1)$. If we adopt a mass ratio distribution that is uniform between 0.6 and 1.0 and zero for $q<0.6$, then the detection thresholds in Figure 4 can be used to predict the number of unidentified companions. The $50 \%$ detection limit spans the full range of possible mass ratios at separations $>10 \mathrm{AU}$, and then it approaches $\Delta m=0$ at a separation of $\sim 4 \mathrm{AU}$. The average threshold of $\Delta m=0.5$ in the separation range $4<a<10 \mathrm{AU}$ corresponds to a detection threshold of $q=0.8$. This implies that all companions outside $10 \mathrm{AU}$ and $1 / 2$ of all companions inside $10 \mathrm{AU}$ were detected. Since we detected one companion within $10 \mathrm{AU}$, we predict the existence of one additional companion, which yields a completeness-corrected binary fraction for separations $\gtrsim 4 \mathrm{AU}$ of $33 \% \pm 17 \%$. If we assume a flat mass ratio distribution between 0.1 and 1.0, as for field stars, then similar analysis finds $1 / 2$ of an undetected companion for separations $10<a<15 \mathrm{AU}$ and two companions for $a \lesssim 10 \mathrm{AU}$, for a complete binary fraction of $46 \% \pm 25 \%$. However, the paucity of observed unequal-mass companions strongly implies that the lower value is more appropriate, and even it might be an overestimate since our Monte Carlo tests produced conservative limits at small separations and USco-109 lies below our nominal detection curve.

We note that the intrinsically higher luminosity of binaries makes them easier to identify in membership surveys, which can bias the binary frequency to larger values. However, this should not significantly bias our results, since all targets except USco-137 are substantially brighter than the detection limit of the original survey by Ardila et al. (2000).

\subsection{Limits on Planetary-Mass Companions}

The high dynamic range observations of young stars and BDs presented here have the potential to directly image wide planetary-mass companions. In Figure 4, we indicate the predicted zero-extinction brightness of some representative masses of planetary companions based on the models of Burrows et al. (1997); since most of our targets have $A_{V}<1$, extinction corrections at $i^{\prime}$ and $z^{\prime}$ should be small $(<0.5 \mathrm{mag})$. The extremely red colors predicted for planetary-mass objects imply that $z^{\prime}$ observations provide the strictest limits on planetary companions. Based on the lack of detections, we conclude that there are no planetary companions with mass $\gtrsim 5 M_{\mathrm{J}}$ at projected separations larger than 280 mas (40 AU) or mass $\gtrsim 10 M_{\mathrm{J}}$ at projected separations larger than 140 mas (20 AU) among our sample. For comparison, these limits would have allowed for marginal detection of the candidate planetary companion to 2MASSW J1207334-393254, a substellar member of the TW Hya association, which has a projected separation of $\sim 54 \mathrm{AU}$, a flux ratio of $\Delta z^{\prime} \sim 7$, and a predicted mass of $\sim 5 M_{\mathrm{J}}$ (Chauvin et al. 2004).

\section{DISCUSSION}

Multiplicity surveys of VLMOs have suggested a fairly uniform set of binary properties in the field (Close et al. 2003; Bouy et al. 2003; Burgasser et al. 2003; Siegler et al. 2005) and in the Pleiades (Martin et al. 2003). No companions with wide separations $(>20 \mathrm{AU})$ or with unequal mass ratios $(q<0.7)$ were found, despite sufficient sensitivity for their detection. The binary fractions observed were also significantly lower than the binary fractions of $57 \%$ for field G dwarfs (Duquennoy \& Mayor 1991 ) and $35 \%-43 \%$ for field M dwarfs (Reid \& Gizis 1997; Fischer \& Marcy 1992). Specifically, Close et al. found a binary fraction of $15 \% \pm 7 \%$ for separations of $\gtrsim 3 \mathrm{AU}$ for field late $\mathrm{M}$ and L dwarfs, Bouy et al. found a binary fraction of $18.8 \% \pm$ $3.7 \%$ at separations of $\gtrsim 1 \mathrm{AU}$ for field $\mathrm{L}$ dwarfs, Burgasser et al. found a binary fraction of $9_{-5}^{+15 \%}$ at separations of $\gtrsim 1 \mathrm{AU}$ for field T dwarfs, Siegler et al. found a binary fraction of $9_{-3}^{+4} \%$ for field M6-M7.5 dwarfs at separations of $\gtrsim 3 \mathrm{AU}$, and Martin et al. found a binary fraction of $15_{-5}^{+15 \%}$ for separations of $\gtrsim 7 \mathrm{AU}$ for Pleiades BDs.

The study presented here is the first survey of young BDs with the resolution and sensitivity to identify binaries as close as $5 \mathrm{AU}$, allowing a more direct comparison with field surveys. Our method of correcting for detection biases is based on that of Close et al. (2003), so our completeness-corrected binary fraction can be compared most directly to that study. Our binary fraction, $33 \% \pm 17 \%$ for separations $\gtrsim 4 \mathrm{AU}$, is higher than, but statistically consistent with, their completeness-corrected binary fraction for field VLMOs $(15 \% \pm 7 \%)$. However, the field surveys are sensitive to companions with separations as small as $\sim 1 \mathrm{AU}$ and found many companions within $4 \mathrm{AU}$ that we could not have detected because of the larger distance to our targets. Our binary fraction for companions with $a \gtrsim 4$ AU only sets a lower limit on the total fraction of VLMO binaries. Higher resolution observations or long-term spectroscopic monitoring of these young 
VLMOs is needed to determine whether they too have an abundance of even closer companions.

All three Upper Sco VLMO binaries have flux ratios of $\Delta m \lesssim$ 1.0 , corresponding to mass ratios of $\gtrsim 0.6$, which also agrees with the other surveys described above, which found no BD binaries with $q<0.6$. The implication is that the mass ratio distribution is not flat at low masses, but instead is biased toward binaries of nearly equal mass. This indicates that a completeness correction that assumes a flat mass ratio for $q \gtrsim 0.1$ would strongly overestimate the VLMO binary frequency. As in the field and open cluster surveys, we found no wide binary companions. Our results are also consistent with a survey of the young ( $3 \mathrm{Myr})$ star-forming region IC 348 by Luhman et al. (2005), which found a $1 \sigma$ upper limit on the binary fraction of $\sim 7 \%$ for separations $>20$ AU. Wide (>20 AU) companions are therefore much less common around VLMOs than around G and early M dwarfs, although some candidate wide companions have been identified in Cha I (Neuhauser et al. 2002; Luhman 2004a) and TW Hya (Chauvin et al. 2004).

The distinct binary properties of field BDs, relative to those of stars, indicate that these properties are mass dependent. However, the form of this dependence is not known; Kroupa et al. (2003) and Close et al. (2003) interpret current data as a break near the stellar/substellar boundary, while Luhman (2004b) argues for a smooth mass dependence. We investigate the mass dependence at a young age by combining our results with a binary census of the more massive T Tauri members of Upper Sco by Kohler et al. (2000). This survey targeted $118 \mathrm{X}$-ray-selected premain-sequence stars ranging from G5 to M5, corresponding to masses of $2.0-0.13 M_{\odot}$, and found a binary fraction of $35.2 \% \pm$ $6.3 \%$ for companions with separations between $6 "$ (1000 AU) and 0 ". 13 (21 AU). Dividing the sample into spectral type bins reveals no spectral type dependence of the binary frequency, although the subsample sizes are not sufficient to completely rule out a gradual decline. Specifically, the survey found a binary frequency of $26 / 50=52 \% \pm 10 \%$ for Upper Sco members of spectral type M0-M5 $\left(0.7-0.13 M_{\odot}\right)$ for separations between 21 and $1000 \mathrm{AU}$, including several very wide companions to primaries in the M4-M5 range. Kohler et al. did not confirm association membership of the companions with colors or proper motions, but they estimate from the low background object density (6.64 \pm $0.45 \mathrm{deg}^{-2}$ in $\mathrm{K}$, comparable to our background object density of $7.8 \pm 0.8 \mathrm{deg}^{-2}$ in $i^{\prime}$ and $z^{\prime}$ ) that no more than $15 \%$ of their detected binaries were spurious claims. They also found no decline in mean or maximum separation and no preference toward equalmass companions with decreasing mass, although the mass ratio result could not be tested for the lowest mass targets since unequalmass companions would have fallen below the detection limit. Our measurements in the mass range $0.04-0.10 M_{\odot}$ suggest a significant decrease in the number of wide binaries. Since none of the newly discovered binaries presented here would have been found in the Kohler survey, there may be a discontinuity in the separation distribution at a mass of $\sim 0.10 M_{\odot}$.

We also note that resolving the newly discovered binaries does not reduce the large scatter in the color-magnitude diagram at low masses that has been observed in previous surveys (Ardila et al. 2000) and is evident for the apparently single VLMOs in Figure 1. Preibisch \& Zinnecker (1999) argue for a consistent age of $5 \mathrm{Myr}$ based on the narrow locus seen on color-magnitude diagrams of more massive Upper Sco members, but the poor fit between our data and any single isochrone of Baraffe et al. (1998) indicates that the lowest mass members of Upper Sco could have a significant range in ages or distances. The uncertainty introduced by transforming the $5 \mathrm{Myr}$ isochrone into the SDSS magnitude system makes it difficult to interpret other possibilities, such as the existence of additional unresolved binaries or preferential selection of older or younger systems in the original survey by Ardila et al.

In summary, the binary properties of the young VLMOs observed here are the first convincing evidence that the small separations and equal-mass ratios of VLMO binaries are established at young ages, rather than as the result of subsequent dynamical evolution after the $\mathrm{T}$ Tauri phase of evolution. The uncertainty in our total binary fraction is too large to directly test the suggestion that the binary fraction declines with declining mass, but if the total binary fraction of the field population is truly primordial, as the small separations and equal masses appear to be, then this provides powerful evidence in support of this assertion. However, complementary spectroscopic surveys are needed to determine whether the apparent deficit of substellar binaries is a true absence or is due to a shift to separations smaller than can be detected with imaging alone. Finally, the recent discovery of candidate wide binary systems in the low-density star-forming regions Cha I (Neuhauser et al. 2002; Luhman 2004a) and TW Hya (Chauvin et al. 2004) indicate that there could be differences in the VLMO binary formation process and initial conditions in OB associations compared to low-density $\mathrm{T}$ associations.

We would like to thank Andrea Ghez for helpful comments, and we thank the anonymous referee for a thorough and helpful response, which improved the quality of this work. This work is based on observations made with the NASA/ESA Hubble Space Telescope, obtained at the Space Telescope Science Institute, which is operated by the Association of Universities for Research in Astronomy, Inc., under NASA contract NAS 5-26555. These observations are associated with program 9853.

\section{REFERENCES}

Ardila, D., Martin, E. L., \& Basri, G. 2000, AJ, 120, 479

Baraffe, I., Chabrier, G., Allard, F., \& Hauschildt, P. H. 1998, A\&A, 337, 403 2002, A\&A, 382, 563

Bouy, H., Brandner, W., Martín, E. L., Delfosse, X., Allard, F., \& Bassri, G. 2003, AJ, 126, 1526

Briceno, C., Luhman, K. L., Hartmann, L., Stauffer, J. R., \& Kirkpatrick, J. D. 2002, ApJ, 580, 317

Bruzual A, G., Persson, S. E., Gunn, J. E., \& Stryker, L. L. 1996, Stellar Atlas (Baltimore: STScI)

Burgasser, A., Kirkpatrick, J. D., Reid, I. N., Brown, M. E., Miskey, C. L., \& Gizis, J. E. 2003, ApJ, 586, 512

Burrows, A., et al. 1997, ApJ, 491, 856

Chauvin, G., Langrange, A.-M., Dumas, C., Zuckerman, B., Mouillet, D., Song, I., Beuzit, J.-L., \& Lowrance, P. 2004, A\&A, 425, L29

Close, L. M., Siegler, N., Freed, M., \& Biller, B. 2003, ApJ, 587, 407
Close, L. M., et al. 2005, Nature, 433, 286

Duquennoy, A., \& Mayor, M. 1991, A\&A, 248, 485

Fischer, D. A., \& Marcy, G. W. 1992, ApJ, 396, 178

Fukugawa, M., Ichikawa, T., Gunn, J. E., Doi, M., Shimasaku, K., \& Schneider, D. P. 1996, AJ, 111, 1748

Gizis, J. E., Reid, I. N., Knapp, G. R., Liebert, J., Kirkpatrick, J. D., Koerner, D. W., \& Burgasser, A. J. 2003, AJ, 125, 3302

Hawley, S. L., et al. 2002, AJ, 123, 3409

Jayawardhana, R., Ardila, D. R., Stelzer, B., \& Haisch, K. E., Jr. 2003, AJ, 126, 1515

Joergens, V., \& Guenther, E. 2001, A\&A, 379, L9

Koerner, D. W., Kirkpatrick, J. D., McElwain, M. W., \& Bonaventura, N. R. 1999, ApJ, 526, L25

Kohler, R., Kunkel, M., Leinert, C., \& Zinnecker, H. 2000, A\&A, 356, 541

Kroupa, P., Bouvier, J., Duchêne, G., \& Moraux, E. 2003, MNRAS, 346, 354 
Liu, W. M., Meyer, M. R., Cotera, A. S., \& Young, E. T. 2003, AJ, 126, 1665 Luhman, K. L. 2004a, ApJ, 614, 398

-. 2004b, ApJ, 617, 1216

Luhman, K. L., McLeod, K. K., \& Goldenson, N. 2005, ApJ, 623, 1141

Luhman, K. L., Stauffer, J. R., Muench, A. A., Rieke, G. H., Lada, E. A., Bouvier, J., \& Lada, C. J. 2003, ApJ, 593, 1093

Mack, J., et al. 2003, ACS Data Handbook, Version 2.0 (Baltimore: STScI)

Martin, E. L., Barrado y Navascués, D., Baraffe, I., Bouy, H., \& Dahm, S. 2003, ApJ, 594, 525

Martin, E. L., Brandner, W., Bouvier, J., Luhman, K. L., Stauffer, J., Basri, G., Zapatero Osorio, M. R., \& Barrado y Navascués, D. 2000, ApJ, 543, 299 Metchev, S. A., Hillenbrand, L. A., \& White, R. J. 2003, ApJ, 582, 1102

Mohanty, S., Jayawardhana, R., \& Basri, G. 2005, ApJ, 626, 498

Muench, A. A., Alves, J., Lada, C. J., \& Lada, E. A. 2001, ApJ, 558, L51
Muzerolle, J., Hillenbrand, L., Calvet, N., Briceño, C., \& Hartmann, L. 2003, ApJ, 592, 266

Neuhauser, R., Brandner, W., Alves, J., Joergens, V., \& Comerón, F. 2002, A\&A, 384, 999

Preibisch, T., \& Zinnecker, H. 1999, AJ, 117, 2381

Reid, I. N., \& Gizis, J. E. 1997, AJ, 113, 2246

Reid, I. N., Gizis, J. E., Kirtkpatrick, J. D., \& Koerner, D. W. 2001, AJ, 121, 489

Reiners, A., Basri, G., \& Mohanty, S. 2005, ApJ, in press

Siegler, N., Close, L. M., Cruz, K. L., Martín, E. L., \& Reid, I. N. 2005, ApJ, 621,1023

Sirianni, M., et al. 2005, PASP, 117, 1049

Stetson, P. B. 1987, PASP, 99, 191

White, R., \& Basri, G. 2003, ApJ, 582, 1109

White, R. J., \& Hillenbrand, L. A. 2004, ApJ, 616, 998 